This is the first drug shown to have a clinically meaningful improvement in type I SMA

\title{
Nusinersen potentially effective in SMA
}

An antisense oligonucleotide, nusinersen, can improve motor function in infants with spinal muscular atrophy (SMA), according to interim results of a phase II study recently published in The Lancet. The study was led by Ionis Pharmaceuticals and conducted by Richard Finkel and colleagues at Nemours Children's Hospital, Stanford University Medical Center, and Columbia University Medical Centre in the USA and the Hospital for Sick Kids in Canada.

SMA is a motor neuron disorder caused by deletions or mutations in the SMN1 gene (encoding survival motor neuron protein, or SMN). The absence of SMN1 in patients is poorly compensated for by its paralogue SMN2, as only $10-25 \%$ of SMN2 transcripts generate full-length SMN. This deficiency is attributable to a variant in the splice site at exon 7 in SMN2. Nusinersen was designed to alter the splicing of SMN2 premRNA to increase the production of full-length SMN, and was well tolerated in a prior phase I safety and dosing study.
This phase II, open-label, doseescalation study included 20 infants with SMA aged between 3 weeks and 7 months, in whom symptom onset had occurred between 3 weeks and 6 months of age. Infants received intrathecal administration of either $6 \mathrm{mg}$ or $12 \mathrm{mg}$ nusinersen on days 1 , 15 and 85 , followed by $12 \mathrm{mg}$ every 4 months thereafter.

In general, nusinersen administration was safe and well tolerated. Although adverse events were observed in all participants, serious events were considered to be diseaserelated and unlikely to be related to nusinersen administration.

The treated infants showed improvements in developmental motor milestones and on a motor test designed to evaluate motor function in infants with SMA. Encouragingly, the trial end point of median age of death or the need for permanent ventilation was not met. In contrast with patients documented in a natural history case series, most of the trial participants survived without permanent ventilation. Four of the 20

participants had died by January 2016.
The researchers were able to evaluate spinal cord tissue from three infants who died during the course of the study. These samples revealed a 2.6-fold increase in full-length SMN2 transcripts in infants who received nusinersen, compared with untreated infants, and an increase in staining intensity of SMN protein in neurons.

"This is the first drug shown to have a clinically meaningful improvement in type I SMA in terms of overall survival, survival free of the requirement for permanent ventilation, and motor function," states Finkel. He concludes that although caution is needed in comparing these open-label data against a historical series, the results were bolstered by positive findings from interim analyses of two phase III trials reported earlier in 2016.

Louise Adams

ORIGINAL ARTICLE Finkel, R. S. et al. Treatment of infantile-onset spinal muscular atrophy with nusinersen: a phase 2, open-label, dose-escalation study. Lancet http://dx.doi.org/10.1016/S01406736(16)31408-8 (2016)

FURTHER READING Faravelli, I. et al. Spinal muscular atrophy - recent therapeutic advances for an old challenge. Nat. Rev. Neurol. 11, 351-359 (2016) 